\title{
Total Synthesis of the Proposed Structure for Spirofungin B: A Reassignment of the Stereochemistry
}

\author{
Shannon D. Zanatta, Jonathan M. White and Mark A. Rizzacasa* \\ School of Chemistry, The University of Melbourne, Victoria 3010, Australia \\ masr@unimelb.edu.au
}

\section{Supporting Information}


General. Unless otherwise stated, ${ }^{1} \mathrm{H}$ NMR $(300 \mathrm{MHz}$ or $400 \mathrm{MHz})$ and proton decoupled ${ }^{13} \mathrm{C}$ NMR spectra (75.5 MHz or $100 \mathrm{MHz}$ ) were recorded for deuterochloroform solutions with residual chloroform as internal standard. Optical rotations were recorded in a $10 \mathrm{~cm}$ microcell. HRMS (ESI) mass spectra were run at Monash University, Clayton, Victoria.

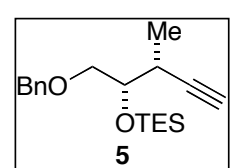

Alkyne 5: $[\square]^{25}=+13.0\left(c, 1.00, \mathrm{CH}_{2} \mathrm{Cl}_{2}\right)$; IR $\square_{\max }$ (film) 3309, 2952, 2910, 2875, $1454 \mathrm{~cm}^{-1} ;{ }^{1} \mathrm{H}$ NMR $(300 \mathrm{MHz}) \square 0.62(\mathrm{q}, J=7.8 \mathrm{~Hz}, 6 \mathrm{H}), 0.95(\mathrm{t}, J=7.8 \mathrm{~Hz}, 9 \mathrm{H})$, $1.20(\mathrm{~d}, J=7 \mathrm{~Hz}, 3 \mathrm{H}), 2.03(\mathrm{~d}, J=2.4 \mathrm{~Hz}, 1 \mathrm{H}), 2.68(\mathrm{ddq}, J=7,6,2.4 \mathrm{~Hz}, 1 \mathrm{H}), 3.52(\mathrm{dd}, J=9.8$, $5.4 \mathrm{~Hz}, 1 \mathrm{H}), 3.61(\mathrm{dd}, J=9.8,4.2 \mathrm{~Hz}, 1 \mathrm{H}), 3.79(\mathrm{ddd}, J=15.4,6,4.2 \mathrm{~Hz}, 1 \mathrm{H}), 4.54(\mathrm{~s}, 2 \mathrm{H}), 7.35-$ 7.26 (m, 5H); ${ }^{13} \mathrm{C}$ NMR (75.5 MHz) $\square 5.0,6.9,16.4,29.6,69.4,72.9,73.3,74.4,86.8,127.5,127.6$, 128.2, 138.3 HRMS (ESI) calcd for $\mathrm{C}_{19} \mathrm{H}_{30} \mathrm{O}_{2} \mathrm{SiNa}\left[\mathrm{M}+\mathrm{Na}^{+}\right]$: 341.1908. Found: 341.1913.

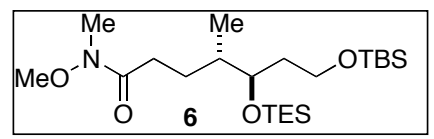

Weinreb amide 6: $[\square]^{25}=+19.3\left(c, 1.00, \mathrm{CH}_{2} \mathrm{Cl}_{2}\right)$; IR $\square_{\max }$ (film) 2954, 2935, 2877, 1674, $1461 \mathrm{~cm}^{-1} ;{ }^{1} \mathrm{H}$ NMR (300 MHz) $\square 0.02(\mathrm{~s}, 6 \mathrm{H})$, 0.57 (q, $J=7.8, \mathrm{~Hz} 6 \mathrm{H}), 0.80-1.07(\mathrm{~m}, 22 \mathrm{H}), 1.42(\mathrm{~m}, 1 \mathrm{H}), 1.49-1.71(\mathrm{~m}, 3 \mathrm{H}), 2.01-2.30(\mathrm{~m}, 2 \mathrm{H})$, $3.16(\mathrm{~s}, 3 \mathrm{H}), 3.66(\mathrm{~s}, 3 \mathrm{H}), 3.72-3.68(\mathrm{~m}, 3 \mathrm{H}) ;{ }^{13} \mathrm{C}$ NMR $(75.5 \mathrm{MHz}) \square-5.6,-5.5,5.0,6.9,14.1,18.2$, $25.7,26.9,27.2,29.8,32.0,34.9,38.3,59.9,60.9,72.0,174.4$; HRMS (ESI) calcd for $\mathrm{C}_{22} \mathrm{H}_{49} \mathrm{NO}_{4} \mathrm{Si}_{2} \mathrm{Na}\left[\mathrm{M}+\mathrm{Na}^{+}\right]$: 470.3098 . Found: 470.3098 .

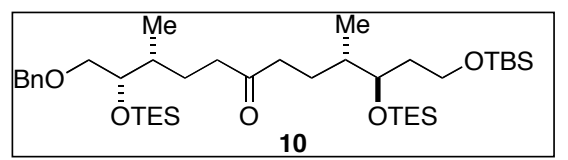

Ketone 10: $[\square]_{\mathrm{D}}^{16}=+11.4\left(c, 2.06, \mathrm{CH}_{2} \mathrm{Cl}_{2}\right)$; IR $\square_{\max }($ film) 2954, 2935, 1717, $14601253 \mathrm{~cm}^{-1} ;{ }^{1} \mathrm{H}$ NMR (300 MHz) $\square 0.04$ $(\mathrm{s}, 6 \mathrm{H}), 0.54-0.62(\mathrm{~m}, 12 \mathrm{H}), 0.83-0.98(\mathrm{~m}, 22 \mathrm{H}), 0.89(\mathrm{~s}, 9 \mathrm{H}), 1.25-1.75(\mathrm{~m}, 10 \mathrm{H}), 2.32-2.48(\mathrm{~m}$, 4H), 3.35-3.45 (m, 2H), 3.63-3.70 (m, 2H), 3.75-3.76, (m, 2H), $4.50(\mathrm{Abq}, J=15 \mathrm{~Hz}, 2 \mathrm{H}), 7.32(\mathrm{~m}$, 5H); ${ }^{13} \mathrm{C}$ NMR (75.5 MHz) $\square-5.34,-5.31,5.10,5.12,6.9,7.0 .13 .8,14.2,18.2,25.3,26.7,27.3$, 
35.2, 36.1, 38.4, 40.8, 41.0, 60.1, 72.2, 73.0, 73.3, 74.4, 127.5, 127.6, 128.3, 138.4, 211.2; HRMS (ESI) calcd for $\mathrm{C}_{39} \mathrm{H}_{76} \mathrm{O}_{5} \mathrm{Si}_{3} \mathrm{Na}\left[\mathrm{M}+\mathrm{Na}^{+}\right]$: 731.4898. Found: 731.4903.

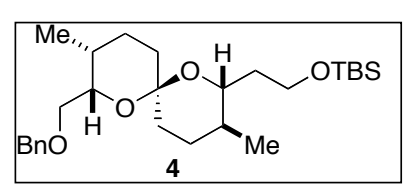

Spiroketal 4: $[\square]_{\mathrm{D}}^{25}=+45\left(c, 0.21, \mathrm{CH}_{2} \mathrm{Cl}_{2}\right) ; \mathrm{IR} \square_{\max }($ film) 2927,2558 , 1497, 1454, $1360 \mathrm{~cm}^{-1} ;{ }^{1} \mathrm{H}$ NMR (300 MHz) $\square 0.05(\mathrm{~s}, 6 \mathrm{H}), 0.81$ (d, $J=6.6$ $\mathrm{Hz}, 3 \mathrm{H}), 0.88(\mathrm{~d}, J=6.9 \mathrm{~Hz}, 3 \mathrm{H}), 0.89(\mathrm{~s}, 9 \mathrm{H}), 1.26-1.61(\mathrm{~m}, 13 \mathrm{H}), 1.85(\mathrm{~m}, 1 \mathrm{H}), 2.07(\mathrm{~m}, 1 \mathrm{H})$, $3.44(\mathrm{~m}, 1 \mathrm{H}), 3.81(\mathrm{~m}, 1 \mathrm{H}), 3.98(\mathrm{td}, J=6,2.4 \mathrm{~Hz}, 1 \mathrm{H}), 4.56(\mathrm{Abq}, J=16 \mathrm{~Hz}, 2 \mathrm{H}), 7.32-7.34(\mathrm{~m}$, $5 \mathrm{H}) ;{ }^{13} \mathrm{C}$ NMR (100 MHz) $\square-5.3,-5.2,1.0,11.2,17.8,18.3,26.0,26.4,28.0,28.1,30.2,34.9,35.7$, $36.7,60.4,70.0,71.6,73.1,95.5,127.3,128.2,138.9 ;$ HRMS (ESI) calcd for $\mathrm{C}_{27} \mathrm{H}_{46} \mathrm{O}_{4} \mathrm{SiNa}\left[\mathrm{M}+\mathrm{Na}^{+}\right]$: 485.3063. Found: 485.3070 .

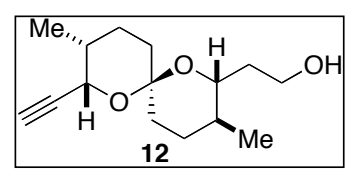

Alcohol 12: $[\square]_{D}^{14}=+51.4\left(c, 0.51, \mathrm{CH}_{2} \mathrm{Cl}_{2}\right)$; IR $\square_{\max }($ film) 3420, 3450, 2950, 2875; ${ }^{1} \mathrm{H}$ NMR (300 MHz) $\square 0.84(\mathrm{~d}, J=6.6 \mathrm{~Hz}, 2 \mathrm{H}), 1.14(\mathrm{~d}, J=6.9 \mathrm{~Hz}$, 2H), 1.35-1.53 (m, 6H), 1.57-1.76 (m, 6H), 1.85-2.04 (m, 3H), $2.43(\mathrm{~d}, J=1.8 \mathrm{~Hz}, 1 \mathrm{H}), 2.88$ (bs, 1H), $3.53(\mathrm{dt}, J=9,2.7 \mathrm{~Hz}, 1 \mathrm{H}), 3.82(\mathrm{t}, J=5.3 \mathrm{~Hz}, 2 \mathrm{H}), 4.57(\mathrm{t}, J=2.3 \mathrm{~Hz}, 1 \mathrm{H}) ;{ }^{13} \mathrm{C} \mathrm{NMR}(100$ MHz) $\square 11.9,17.6,25.1,27.3,29.2,31.1,34.6,34.7,35.5,61.5,63.3,73.1,76.4,82.7,96.6$; HRMS (ESI) calcd for $\mathrm{C}_{15} \mathrm{H}_{24} \mathrm{O}_{3} \mathrm{Na}\left[\mathrm{M}+\mathrm{Na}^{+}\right]$: 275.1623 Found 275.1620.

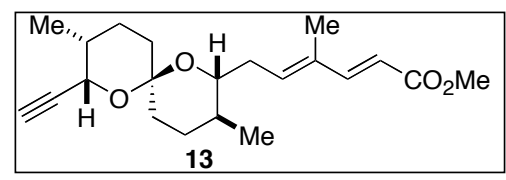

Ester 13: $[\square]_{D}^{14}=-62.1\left(c, 1.13, \mathrm{CH}_{2} \mathrm{Cl}_{2}\right)$; IR $\square_{\max }($ film) 2933, $2873,1717,1622,1434 \mathrm{~cm}^{-1} ;{ }^{1} \mathrm{H}$ NMR (300 MHz) 0.86 (d, $J=6.6$ $\mathrm{Hz}, 3 \mathrm{H}), 1.11(\mathrm{~d}, J=7.2 \mathrm{~Hz}, 3 \mathrm{H}), 1.29-1.49(\mathrm{~m}, 6 \mathrm{H}), 1.55-1.73(\mathrm{~m}, 5 \mathrm{H}), 1.79-1.87(\mathrm{~m}, 4 \mathrm{H}), 1.92-$ $2.03(\mathrm{~m}, 1 \mathrm{H}), 2.30(\mathrm{~m}, 1 \mathrm{H}), 2.40(\mathrm{~d}, J=2.4 \mathrm{~Hz}, 1 \mathrm{H}), 2.52(\mathrm{~m}, 1 \mathrm{H}), 3.35(\mathrm{dt}, J=9,3 \mathrm{~Hz}, 1 \mathrm{H}), 3.74$ $(\mathrm{s}, 3 \mathrm{H}), 4.45(\mathrm{t}, J=2.4 \mathrm{~Hz}, 1 \mathrm{H}), 5.80(\mathrm{~d}, J=15.6 \mathrm{~Hz}, 1 \mathrm{H}), 6.06(\mathrm{t}, J=7.2 \mathrm{~Hz}, 1 \mathrm{H}), 7.35(\mathrm{~d}, J=15.6$ $\mathrm{Hz}, 1 \mathrm{H}) ;{ }^{13} \mathrm{C}$ NMR (100 MHz) 12.0, 12.4, 17.6, 24.9, 27.6, 29.3, 31.1, 32.4, 34.6, 35.4, 51.4, 63.1, 72.9, 74.5, 82.9, 96.5, 115.1, 134.1, 138.6, 149.7, 168.1; HRMS (ESI) calcd for $\mathrm{C}_{21} \mathrm{H}_{33} \mathrm{O}_{4} \mathrm{Na}\left[\mathrm{M}+\mathrm{Na}^{+}\right]:$369.2042. Found: 369.2040. 


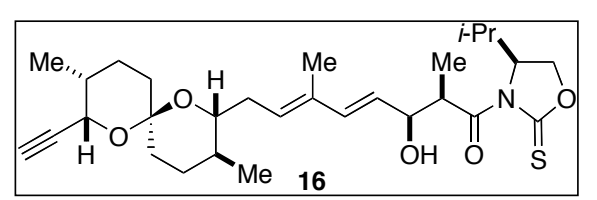

Aldol adduct 16: $[\square]_{D}^{16}=+48.8\left(c, 0.15, \mathrm{CH}_{2} \mathrm{Cl}_{2}\right)$; IR $\square_{\max }$ (film) 3450, 3286, 2929, 2873, 1697, 1452; ${ }^{1} \mathrm{H}$ NMR (400

$\mathrm{MHz}) \square 0.86(\mathrm{~d}, J=6.4 \mathrm{~Hz}, 3 \mathrm{H}), 0.88(\mathrm{~d}, J=6.8 \mathrm{~Hz}, 3 \mathrm{H}), 0.93(\mathrm{~d}, J=7.2 \mathrm{~Hz}, 3 \mathrm{H}), 1.12(\mathrm{~d}, J=$ $7.2 \mathrm{~Hz}, 3 \mathrm{H}), 1.19$ (d, $J=6.8 \mathrm{~Hz}, 3 \mathrm{H}), 1.34-1.49$ (m, 4H), 1.56-1.73 (m, 4H) 1.77 (s, 3H), 1.79-1.85 (m, 2H), $2.03(\mathrm{~m}, 1 \mathrm{H}), 2.26(\mathrm{~m}, 1 \mathrm{H}), 2.44(\mathrm{~m}, 1 \mathrm{H}), 2.40(\mathrm{~d}, J=2.0 \mathrm{~Hz}, 1 \mathrm{H}), 2.64(\mathrm{~d}, J=3.2 \mathrm{~Hz}$, 1H), $3.31(\mathrm{ddd}, J=12,8,3.2 \mathrm{~Hz}, 1 \mathrm{H}), 4.38(\mathrm{~d}, J=6 \mathrm{~Hz}, 2 \mathrm{H}), 4.51(\mathrm{t}, J=2.4 \mathrm{~Hz}, 1 \mathrm{H}), 4.68(\mathrm{~m}$, 1H), $4.77(\mathrm{~m}, 1 \mathrm{H}), 5.04(\mathrm{~m}, 1 \mathrm{H}), 5.61(\mathrm{dd}, J=15.8,6.4 \mathrm{~Hz}, 1 \mathrm{H}), 5.67(\mathrm{t}, J=7.2 \mathrm{~Hz}, 1 \mathrm{H}), 6.63(\mathrm{~d}, J$ $=15.8 \mathrm{~Hz}, 1 \mathrm{H}) ;{ }^{13} \mathrm{C} \mathrm{NMR}(100 \mathrm{MHz}) \square 11.2,12.0,12.7,14.9,17.7,18.3,25.0,27.8,29.1,29.4$, $31.3,31.8,34.5,35.4,42.7,63.0,63.3,67.4,72.8,73.5,74.9,83.1,96.5,125.2,130.0,134.2,136.7$, 176.8, 186.1; HRMS (ESI) calcd for $\mathrm{C}_{29} \mathrm{H}_{43} \mathrm{NNaO}_{5} \mathrm{NaS}\left[\mathrm{M}+\mathrm{Na}^{+}\right]$: 540.2760. Found: 540.2758.

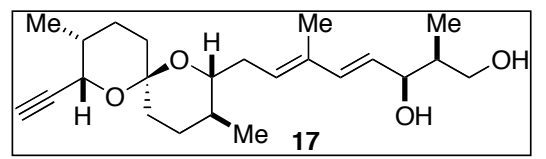

Diol 17: $[\square]^{23}=-42.3\left(c, 0.14, \mathrm{CH}_{2} \mathrm{Cl}_{2}\right)$; IR $\square_{\max }($ film) 3391, $3311,2931,1655,1459 \mathrm{~cm}^{-1} ;{ }^{1} \mathrm{H}$ NMR $(400 \mathrm{MHz}) \square 0.86-0.91(\mathrm{~m}$, $6 \mathrm{H}), 1.13(\mathrm{~d}, J=7.2 \mathrm{~Hz}, 3 \mathrm{H}), 1.44-1.49(\mathrm{~m}, 3 \mathrm{H}), 1.34-1.42(\mathrm{~m}, 3 \mathrm{H}) 1.54-1.86(\mathrm{~m}, 6 \mathrm{H}), 1.97-2.07$ (m, 2H), 2.17-2.29 (m, 2H), $2.40(\mathrm{~d}, J=2 \mathrm{~Hz}, 1 \mathrm{H}), 2.45-2.50(\mathrm{~m}, 2 \mathrm{H}), 3.64-3.74(\mathrm{~m}, 2 \mathrm{H}), 3.32(\mathrm{~m}$, $1 \mathrm{H}), \quad 4.36(\mathrm{~m}, 1 \mathrm{H}) 4.51(\mathrm{~m}, 1 \mathrm{H}), 5.62-5.69(\mathrm{~m}, 2 \mathrm{H}), 6.31(\mathrm{~d}, J=15.6 \mathrm{~Hz}, 1 \mathrm{H}) ;{ }^{13} \mathrm{C} \mathrm{NMR}(100$ $\mathrm{MHz}) \square 11.6,12.0,12.8,17.7,25.0,27.8,29.4,31.3,31.8,34.5,35.4,40.2,63.0,66.4,72.8,75.0$, 76.5, 83.1, 96.5, 126.2, 129.9, 134.2, 136.6; HRMS (ESI) calcd for $\mathrm{C}_{23} \mathrm{H}_{36} \mathrm{O}_{4} \mathrm{Na}\left[\mathrm{M}+\mathrm{Na}^{+}\right]: 399.2511$. Found: 399.2516.

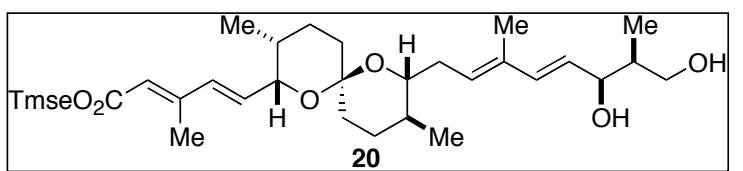

Alcohol 20: $[\square]^{23}=-37.0\left(c, 0.02, \mathrm{CH}_{2} \mathrm{Cl}_{2}\right) ;$ IR $\square_{\max }$ (film) 3422, 2954, 2928, 1709,1560; ${ }^{1} \mathrm{H}$ NMR (400 $\mathrm{MHz}) \square 0.05(\mathrm{~s}, 9 \mathrm{H}), 0.84-0.94$ (m, 6H), 1.00-1.07 (m, 3H), 1.25-1.67 (m, 13H), 1.76 (s, 3H), 1.97$2.31(\mathrm{~m}, 3 \mathrm{H}), 2.28(\mathrm{~s}, 3 \mathrm{H}), 2.44-2.49(\mathrm{~m}, 1 \mathrm{H}), 3.18(\mathrm{td}, J=8,3.2 \mathrm{~Hz}, 1 \mathrm{H}), 3.66-3.74(\mathrm{~m}, 2 \mathrm{H}), 4.19-$ $4.23(\mathrm{~m}, 2 \mathrm{H}), 4.33(\mathrm{~m}, 1 \mathrm{H}), 4.38(\mathrm{~m}, 1 \mathrm{H}), 5.63-5.71(\mathrm{~m}, 2 \mathrm{H}), 5.74(\mathrm{~s}, 1 \mathrm{H}), 6.03(\mathrm{dd}, J=15.8,5 \mathrm{~Hz}$, $1 \mathrm{H}), 6.25(\mathrm{~d}, J=15.8 \mathrm{~Hz}, 1 \mathrm{H}), 6.32(\mathrm{~d}, J=15.8 \mathrm{~Hz}, 1 \mathrm{H}) ;{ }^{13} \mathrm{C}$ NMR $(100 \mathrm{MHz}) \square-1.5,11.5,11.6$, 
12.7, 13.8, 17.4, 17.8, 26.2, 27.8, 28.0, 29.7, 31.5, 31.8, 34.7, 35.67, 40.4, 61.9, 66.4, 71.1, 75.0, $76.3,76.7,96.1,119.1,126.1,130.3,132.4,133.9,136.6,167.4$; HRMS (ESI) calcd for $\mathrm{C}_{32} \mathrm{H}_{54} \mathrm{O}_{6} \mathrm{SiNa}\left[\mathrm{M}+\mathrm{Na}^{+}\right]:$585.3587. Found: 585.3596.

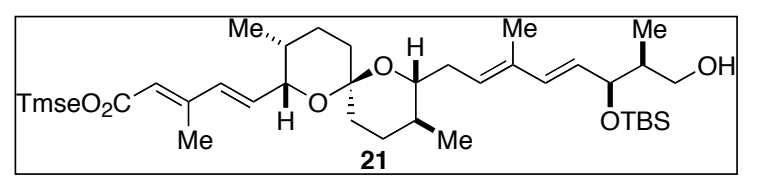

TBS Ether 21: $[\square]^{20}{ }_{D}=-129.9\left(c, 0.14, \mathrm{CH}_{2} \mathrm{Cl}_{2}\right)$ IR (film) 3422, 2954, 2928, 1709,1560; ${ }^{1} \mathrm{H}$ NMR $(400 \mathrm{MHz}) \square 0.02(\mathrm{~s}, 3 \mathrm{H}), 0.05(\mathrm{~s}, 9 \mathrm{H}), 0.08(\mathrm{~s}, 3 \mathrm{H}), 0.80(\mathrm{~d}, J=6.8 \mathrm{~Hz}, 3 \mathrm{H}), 0.88-0.85(\mathrm{~m}, 6 \mathrm{H})$, $0.90(\mathrm{~s}, 9 \mathrm{H}), 1.03-0.99(\mathrm{~m}, 3 \mathrm{H}), 1.25-1.71(\mathrm{~m}, 8 \mathrm{H}), 1.75(\mathrm{~s}, 3 \mathrm{H}), 2.00-2.20(\mathrm{~m}, 3 \mathrm{H}), 2.28(\mathrm{~s}, 3 \mathrm{H})$, $2.48(\mathrm{~m}, 1 \mathrm{H}), 2.89(\mathrm{bs}, 1 \mathrm{H}), 3.16(\mathrm{td}, J=8,2.6 \mathrm{~Hz}, 1 \mathrm{H}), 3.49(\mathrm{~m}, 1 \mathrm{H}), 3.67(\mathrm{~m}, 1 \mathrm{H}), 4.21(\mathrm{~m}, 1 \mathrm{H})$, $4.33(\mathrm{~m}, 1 \mathrm{H}), 5.59(\mathrm{dd}, \mathrm{J}=15.4,6.8 \mathrm{~Hz}, 1 \mathrm{H}), 5.65(\mathrm{t}, J=7.6 \mathrm{~Hz}, 1 \mathrm{H}), 5.74(\mathrm{~s}, 1 \mathrm{H}), 6.01(\mathrm{dd}, J=$ 15.4, $5 \mathrm{~Hz}, 1 \mathrm{H}), 6.23(\mathrm{~d}, J=15.4 \mathrm{~Hz}, 1 \mathrm{H}), 6.23(\mathrm{~d}, J=15.4 \mathrm{~Hz}, 1 \mathrm{H}) ;{ }^{13} \mathrm{C} \mathrm{NMR}(100 \mathrm{MHz}) \square-5.1,-$ $4.3,-1.5,11.5,12.5,12.7,13.8,17.4,17.8,18.1,25.8,26.2,28.1,29.7,31.5,31.8,35.0,35.7,41.3$, $61.9,65.9,71.1,75.1,77.6,96.1,119.1,125.7,129.9,132.4,134.0,136.3,136.5,151.9,167.3$; HRMS (ESI) calcd for $\mathrm{C}_{44} \mathrm{H}_{92} \mathrm{O}_{6} \mathrm{Si}_{3} \mathrm{Na}\left[\mathrm{M}+\mathrm{Na}^{+}\right]$: 699.4452. Found: 699.4442.

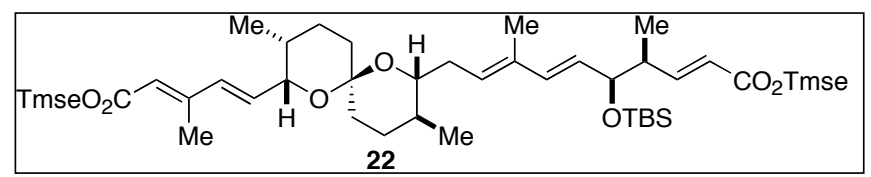

Diester 22: $[\square]^{23}{ }_{D}=-44.0\left(c, 0.14, \mathrm{CH}_{2} \mathrm{Cl}_{2}\right)$ IR $\square_{\max }\left(\right.$ film) 2954, 2927, 2856, 1714, 1614; ${ }^{1} \mathrm{H}$ NMR (400 MHz) \-0.02 (s, 3H), 0.03 (s, 3H), 0.04 (s, 9H), 0.05 (s, 9H), 0.84-0.89 (m, 15H), 0.98$1.03(\mathrm{~m}, 8 \mathrm{H}), 1.25-1.42(\mathrm{~m}, 10 \mathrm{H}), 1.46-1.70(\mathrm{~m}, 22 \mathrm{H}), 1.72(\mathrm{~s}, 3 \mathrm{H}), 2.10(\mathrm{~m}, 1 \mathrm{H}), 2.28(\mathrm{~s}, 3 \mathrm{H}), 2.45$ $(\mathrm{m}, 1 \mathrm{H}), 3.16(\mathrm{dt}, J=8,2.8 \mathrm{~Hz}, 1 \mathrm{H}), 4.14(\mathrm{~m}, 1 \mathrm{H}), 4.19-4.24(\mathrm{~m}, 5 \mathrm{H}), 4.31(\mathrm{~m}, 1 \mathrm{H}), 5.46(\mathrm{dd}, J=$ 15.6, $6.8 \mathrm{~Hz}, 1 \mathrm{H}), 5.62(\mathrm{t}, J=7 \mathrm{~Hz}, 1 \mathrm{H}), 5.74(\mathrm{~s}, 1 \mathrm{H}) 5.77(\mathrm{~d}, J=15.6 \mathrm{~Hz}, 1 \mathrm{H}), 6.02(\mathrm{dd}, J=15.6$, $5 \mathrm{~Hz}, 1 \mathrm{H}), 6.19(\mathrm{~d}, J=15.6 \mathrm{~Hz}, 1 \mathrm{H}), 6.24(\mathrm{~d}, J=15.6 \mathrm{~Hz}, 1 \mathrm{H}), 6.99(\mathrm{dd}, J=15.6,7 \mathrm{~Hz}, 1 \mathrm{H}) ;{ }^{13} \mathrm{C}$ NMR (100 MHz) $\square-4.9,-4.1,-1.5,11.5,12.7,13.7 .14 .0,17.3,17.4,17.8,18.2,23.7,25.9,26.1$, $28.1,29.7,31.5,31.8,34.9,35.7,43.6,61.8,62.3,71.0,75.0,76.5,77.2,96.1,119.1,121.1,127.1$, $129.6,132.4,134.0,135.9,136.6,151.4,151.9,166.9,167.4$; HRMS (ESI) calcd for $\mathrm{C}_{44} \mathrm{H}_{80} \mathrm{O}_{6} \mathrm{Si}_{3} \mathrm{Na}\left[\mathrm{M}+\mathrm{Na}^{+}\right]: 839.5110$. Found: 839.5114. 


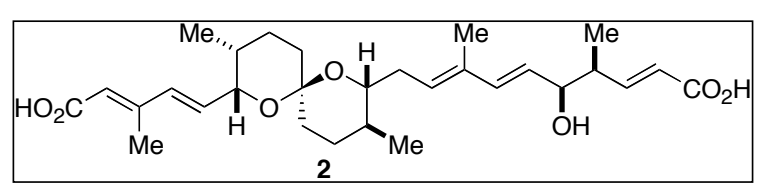

Diacid 2: $[\square]_{D}^{24}=-19.2\left(\mathrm{c}, 0.11, \mathrm{CH}_{2} \mathrm{Cl}_{2}\right) ; \mathrm{IR}_{\max }$ (film) 2924, 2855, 2595, 2342, 1694, 1682; ${ }^{1} \mathrm{H}$ NMR $(400 \mathrm{MHz}) \square 0.87(\mathrm{~d}, J=6.8 \mathrm{~Hz}, 3 \mathrm{H}), 0.90(\mathrm{~d}, J=7.2 \mathrm{~Hz}, 3 \mathrm{H}), 1.08(\mathrm{~d}, J=6.8 \mathrm{~Hz}, 3 \mathrm{H}), 1.11-1.71$ (m, 12H), $1.73(\mathrm{~s}, 3 \mathrm{H}), 2.09(\mathrm{~m}, 1 \mathrm{H}), 2.28(\mathrm{~s}, 3 \mathrm{H}), 2.51(\mathrm{~m}, 1 \mathrm{H}), 2.63(\mathrm{~m}, 1 \mathrm{H}), 3.16(\mathrm{dt}, J=12,2.4$ $\mathrm{Hz}, 1 \mathrm{H}), 3.29(\mathrm{bs}, 1 \mathrm{H}), 4.17(\mathrm{~m}, 1 \mathrm{H}), 4.30(\mathrm{~m}, 1 \mathrm{H}), 5.51(\mathrm{dd}, J=15.8,7.6 \mathrm{~Hz} 1 \mathrm{H}), 5.74(\mathrm{t}, J=7.2$ $\mathrm{Hz}, 1 \mathrm{H}), 5.78(\mathrm{~s}, 1 \mathrm{H}), 5.87(\mathrm{~d}, J=15.8 \mathrm{~Hz}, 1 \mathrm{H}), 6.08(\mathrm{dd}, J=15.8,5.6 \mathrm{~Hz}, 1 \mathrm{H}), 6.23(\mathrm{~d}, J=15.8$ $\mathrm{Hz}, 1 \mathrm{H}), 6.29(\mathrm{~d}, J=15.8 \mathrm{~Hz}, 1 \mathrm{H}), 7.10(\mathrm{dd}, J=15.8,7.6 \mathrm{~Hz}, 1 \mathrm{H}) ;{ }^{13} \mathrm{C} \mathrm{NMR}(100 \mathrm{MHz}) \square 11.5$, $12.6,14.0,14.9,17.7,22.7,26.3,28.1,31.5,31.7,35.2,42.6,71.4,75.4,77.2,96.2,118.3,121.1$, 125.6, 131.5, 132.9, 133.6, 137.3, 138.0, 152.5, 154.8, 171.2, 172.1; HRMS (ESI) calcd for $\mathrm{C}_{29} \mathrm{H}_{42} \mathrm{NaO}_{7}\left[\mathrm{M}+\mathrm{Na}^{+}\right]:$525.2828. Found: 525.2827. 

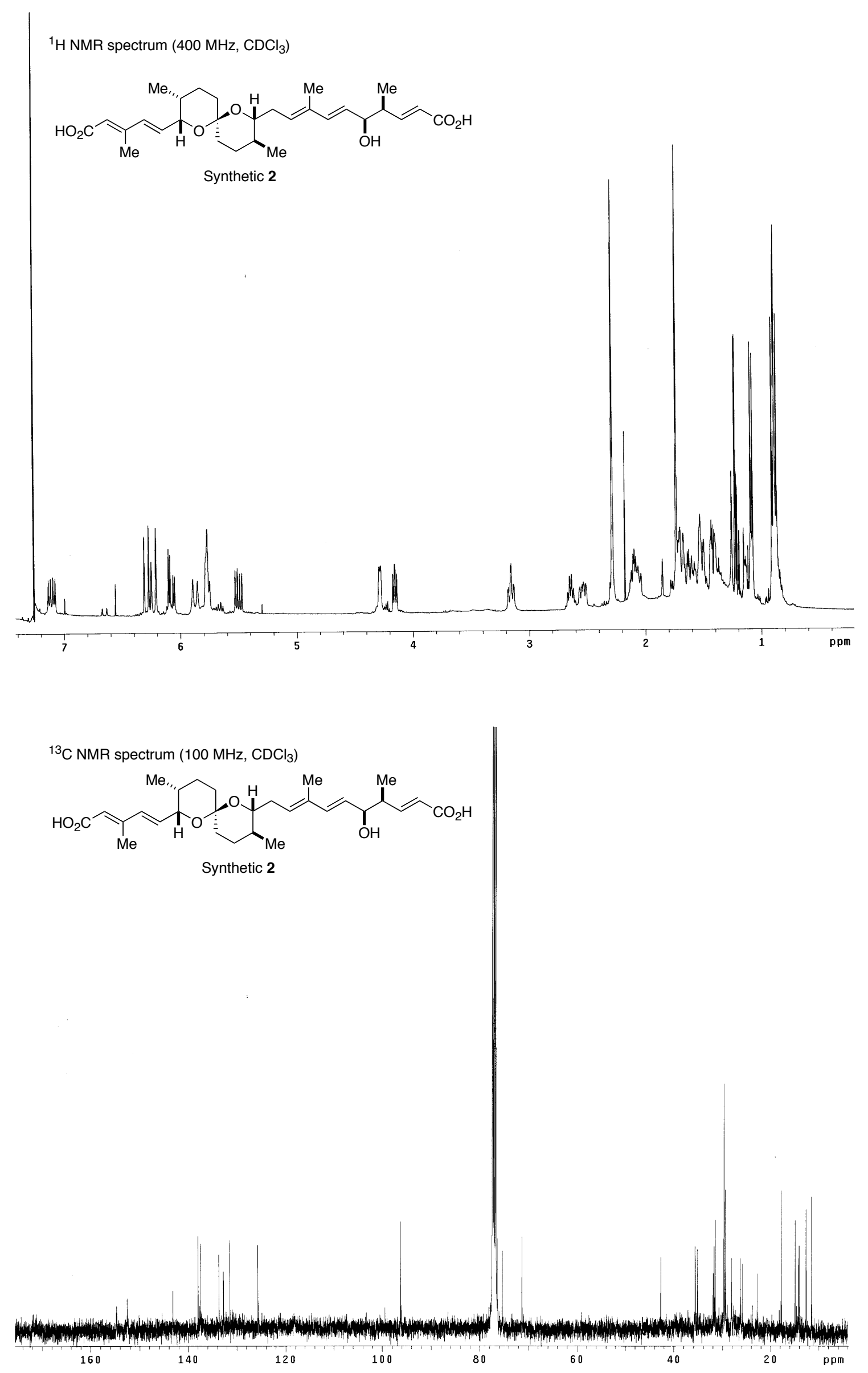
${ }^{1} \mathrm{H}$ NMR spectrum $\left(400 \mathrm{MHz}, \mathrm{CDCl}_{3}\right)$

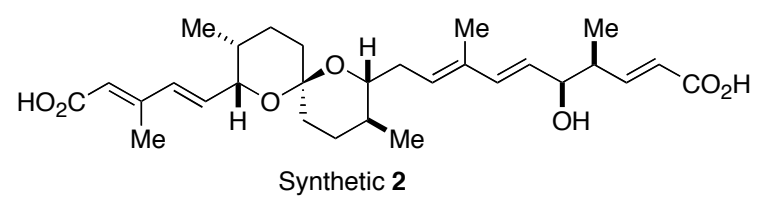

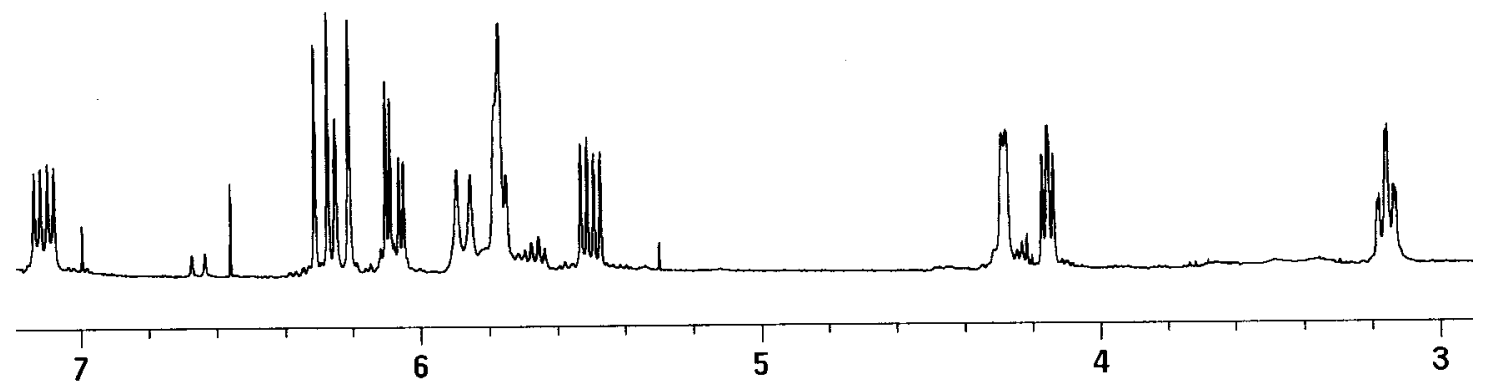

${ }^{1} \mathrm{H}$ NMR spectrum $\left(400 \mathrm{MHz}, \mathrm{CDCl}_{3}\right.$ ) Spirofungins A \& B: Authentic Sample (4:1 mixture)

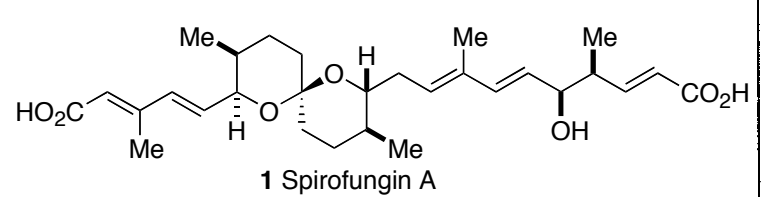

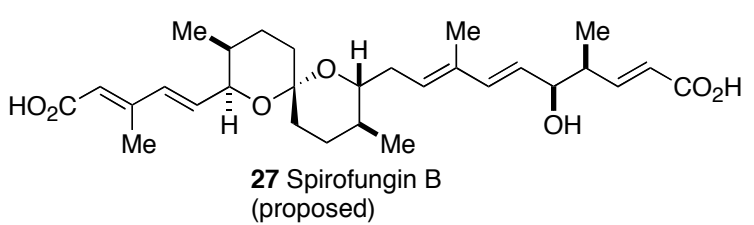
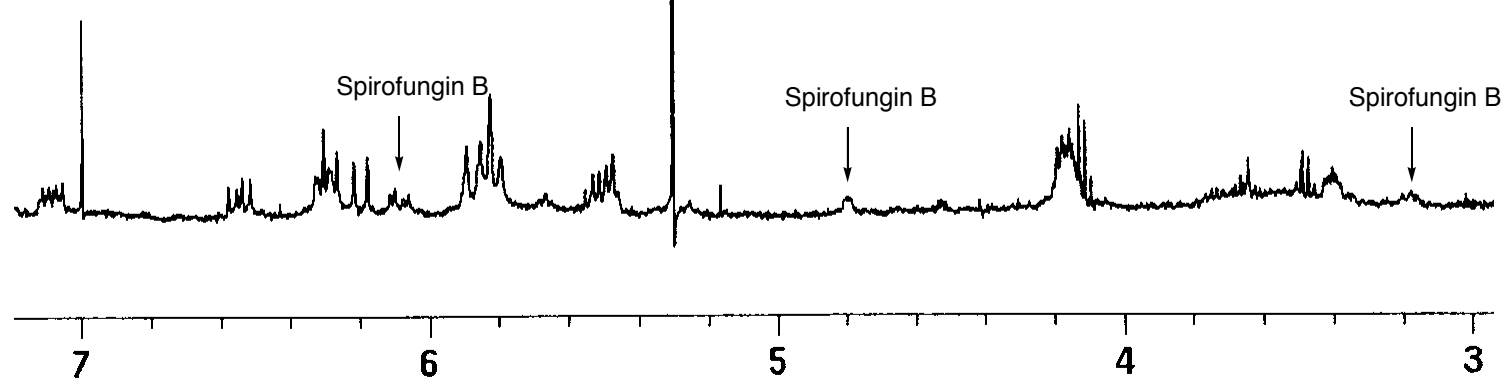